\title{
Investigating Parameter Interactions with the Factorial Design Method: Webster's Optimal Cycle Length Model
}

\author{
Ali Payıdar AKGÜNGÖR, Ersin KORKMAZ
}

\begin{abstract}
Accurate estimation of cycle length is an important factor in the performance of a signalized intersection. Cycle length is determined by employing some parameters such as arrival flow, number of phase, lost time etc., but each parameter has different effects on a cycle length model. If the effects of parameters and their interactions in the cycle length model are known, the performance of the model can be effectively increased. In this study, the sensitivity of optimal cycle length model proposed by Webster and its parameters were analysed with the factorial design method. The reason for selecting this model is that the model has still been used in signal timing practice and has lead many studies of researchers over 50 years. The evaluation of sensitivity analysis shows that while arrival flow as single parameter has a major effect on the optimal cycle length model, the remaining single parameters of the model (i.e., the number of phase in a cycle length, saturation flow and lost time) have secondary importance. Additionally, two parameter interactions of arrival flow-saturation flow have major effect on the model results. For three parameter interactions, the number of phase-arrival flow-saturation flow interaction has a slightly larger effect than the other three-parameter interactions. As a result, the factorial design method is an effective tool to determine the importance of the model parameters for researchers, and it can be employed to other traffic engineering applications.
\end{abstract}

Keywords: arrival flow; factorial design method; optimal cycle length; sensitivity analysis; Webster model

\section{INTRODUCTION}

Sensitivity analysis of a model is useful to determine relative effects of model parameters on model results. The one-way sensitivity analysis, named as change of onefactor-at-a-time, is a widely used sensitivity analysis because of its simplicity. In this method, only one parameter is changed at one time by a given amount, and is examined for the impact that the change has on the model's results. On the other hand, the major weakness of this method is its inability to identify multiple factor interactions among the model parameters. As an alternative approach, the factorial design method developed by Box et al. has been successfully employed in various sensitivity studies [1-5]. Unlike the standard "change one-factor-at-atime" sensitivity approach, this method has the advantage of testing both the sensitivity of model results to changes in individual parameters, and to interactions within a group of parameters.

This method has successfully been applied to different models in traffic and transportation engineering. Akgüngör et al. investigated the parameter effects of HCM 2000 delay model by factorial design method. This study showed that the arrival flow, the saturation flow, and the green signal time are the main parameters that significantly affect the average control delay. Additionally, the multi-parameter interactions of the arrival flow-saturation flow and the arrival flow-green signal time have major effects on the model. This study also illustrated that the effect of parameters on the uniform delay showed that the green signal time and the cycle length appeared to significantly affect the uniform delay [6]. Akgüngör and Yıldız applied fractional factorial method to an accident prediction model. The evaluation of sensitivity analysis indicated that average daily traffic (ADT), lane width (W), width of paved shoulder (PA), median $(\mathrm{H})$ and their interactions (i.e., ADT-W, ADT-PA and ADT-H) have significant effects on number of accidents. Based on the absolute value of parameter effects at the three- and two-standard deviation thresholds ADT was found to be of primary importance, while the remaining identified parameters seemed to be of secondary importance [7]. Akgüngör performed another study with factorial design method to determine the parameter effects of Webster delay model. The study results showed that the cycle length and green time are the most effective two parameters for the delay model. Moreover, the cycle length - green time and traffic volume- cycle length have major effects for the multiparameter interactions. Delay studies with factorial design method investigated by Akgüngör showed that cycle length is an important parameter for intersection performance [8]. Average vehicle delay at a signalized intersection can be minimized by a good estimate of the cycle length. Longer and shorter cycle lengths cause increased delay and queues. In fact, short cycle lengths do not provide adequate green time for all phases and result in cycle failures, while longer cycle lengths cause unused green time. This forces vehicles to wait longer than necessary, and results in more waiting time and queues. Therefore, estimation of optimal cycle length is the most important parameter for traffic signal system. Additionally, capacity, delay, start-up lost time are other important parameters for traffic signal system and they are related to optimal cycle length [9-11]. Çalışkenlli et al. [12] showed that start-up lost time is a significant parameter and it increases rapidly as cycle time increases.

Over the past 50 years, many cycle length models have been developed by researchers for optimal signal operations. One of the oldest models presented in the literature is optimal cycle length model derived by Webster in 1958 [13]. After Webster's optimal cycle length expression, more representative models have been proposed by many researchers using various techniques. Miller [14] developed a formula for optimum cycle time to minimize the overall average delay. He used the lowest of the saturation flows for any of the representative movements. Lan [15] used cycle length and traffic flow parameters including duration of analysis period in his model and Lan and $\mathrm{Gu}$ [16] proposed a new formulation for optimal cycle length by employing non-linear regression analysis. Cheng et al. [17, 18] modified Webster's optimal cycle length equation based on HCM 2000 and suggested a quick estimation method. 
The individual affects and interactions between the parameters in the experiment may be determined by the factorial design method. Thus, the experiment results are obtained in a shorter time with fewer experiments. However, this study is not an experimental design and is different from the studies in the literature. In here, the factorial design method has been employed to determine the sensitivity of Webster optimal cycle length model widely used in traffic signalization. The reason for selecting this model is that it has still been used in signal timing practice and lead many studies of researchers over the past 50 years. In the light of this study, novel cycle length models are developed for future studies after determining effective parameters. Thus, this study shows that the factorial design method can be used for different purposes besides experiment design. Additionally, determining the effective parameters on cycle length models by the factorial design method is important for traffic researches and traffic signals practitioners.

This paper is organized in the following way. In section 2, the factorial design method is explained. The application of Webster optimal cycle length model by the factorial design method is presented in Section 3. Section 4 is devoted to results and discussions. Finally, conclusions are given in the last section.

\section{FACTORIAL DESIGN METHOD}

This method is used to examine the sensitivity of changes in singular parameters and interactions between groups of parameters as well. When the number of the parameters is equal to five or less a full factorial design can be carried out. In this method, a fixed number of possible values for each of the model parameters is tested considering specific perturbations of the magnitudes of the parameters (usually two levels: upper and lower) assigned before. Then, each parameter is identified and ranked according to some pre-established measure of model sensitivity by running the model through all possible combinations of the parameters. For instance, a factorial design at two levels for $\mathrm{k}$ parameters will result in $2^{k}$ combinations of the model parameters. This is explained in the following 3-parameter ( $2^{3}$ factorial) design with parameters $\omega, \psi$ and $\gamma$, and prediction variable $R$. Here, "+" and "-" signs represent the two possible values of each parameter (upper and lower levels, respectively). The effects of parameters and parameter interactions on the model results are predicted by utilizing Eq. (1) and the factorial design matrix given in Tab. 1. The signs of the parameter interactions are determined by the following rule: plus times minus produces a minus, and minus times minus or plus times plus produces a plus. According to this rule, the corresponding computation matrix for parameter interactions is given in Tab. 2.

$$
E_{j}=\sum_{i=1}^{n} \frac{S_{i j} R_{i}}{N_{j}}
$$

in which $E_{j}$ indicates the effect of the $j^{\text {th }}$ factor, $n$ represents the total number of experimental runs, $S_{i j}$ illustrates the sign in row $i$ and column $j, R_{i}$ describes the value of the prediction variable obtained from the $i^{\text {th }}$ experimental run and $N_{j}$ shows the number of "+" signs in column $j$.

Table 1 Design matrix for single parameters

\begin{tabular}{|c|c|c|c|c|}
\hline & \multicolumn{3}{|c|}{ Design Matrix } & Prediction Variable \\
\hline Run & $\omega$ & $\psi$ & $\gamma$ & $\mathrm{R}$ \\
\hline 1 & - & - & - & $\mathrm{R} 1$ \\
\hline 2 & + & - & - & $\mathrm{R} 2$ \\
\hline 3 & - & + & - & $\mathrm{R} 3$ \\
\hline 4 & + & + & - & $\mathrm{R} 4$ \\
\hline 5 & - & - & + & $\mathrm{R} 5$ \\
\hline 6 & + & - & + & $\mathrm{R} 6$ \\
\hline 7 & - & + & + & $\mathrm{R} 7$ \\
\hline 8 & + & + & + & $\mathrm{R} 8$ \\
\hline
\end{tabular}

Table 2 Computation matrix for the three-parameter interactions

\begin{tabular}{|c|c|c|c|c|}
\hline & \multicolumn{5}{|c|}{ Computation Matrix } \\
\hline Run & $\omega \cdot \psi$ & $\omega \cdot \gamma$ & $\psi \cdot \gamma$ & $\omega \cdot \psi \cdot \gamma$ \\
\hline 1 & + & + & + & - \\
\hline 2 & - & - & + & + \\
\hline 3 & - & + & - & + \\
\hline 4 & + & - & - & - \\
\hline 5 & + & - & - & + \\
\hline 6 & - & + & - & - \\
\hline 7 & - & - & + & - \\
\hline 8 & + & + & + & + \\
\hline
\end{tabular}

After all, $E_{j}$ values are computed by Eq. (1), the degree of importance of the parameters and their interactions can be evaluated. As recommended by Box et al. [1] the parameters with major effects are identified and ranked by plotting the effects on a normal probability scale. In this method, any outliers from the straight line on this probability paper could be regarded to influence the model results significantly. On the other hand, the other effects would result in variability in model results consistent with the result of random variation about a fixed mean. Here, it is assumed that higher order interactions are insignificant, in a manner similar to neglecting higher order terms in a Taylor series expansion. Henderson-Sellers [2-3] suggested another method to identify the parameters that have major effects on the model results. According to this method, thresholds are obtained two, three or four standard deviations from zero. Thus, any effects greater than the predicted thresholds are assumed to have significant effects on the model results.

\section{APPLICATION OF THE FACTORIAL DESIGN METHOD FOR WEBSTER'S OPTIMAL CYCLE LENGTH MODEL}

A cycle length is a time required to complete the movements in the phases at a signalized intersection. The optimal cycle length provides sufficient capacity and minimum delay. Cycle lengths usually range between 40 and 150 seconds according to traffic flow and the number of the phase. On the other hand, short and long cycle lengths significantly cause the increase in delay.

Traffic engineers and researchers have used Webster's principles and expressions to determine the optimal cycle length and green split allocation at isolated intersections, regulated by traffic signals with a pre-timed control. Webster's model has never lost its popularity for long decades since his principles are straightforward and easy. When developing his model, Webster accepted the following two principles for signal timing strategies. First, for a given cycle length, fixed time signals should have 
their critical phase time for equal degrees of saturation. Second, effective green times of the phases are in the ratio of their respective flow ratio values. The optimal cycle length model proposed by Webster can be expressed as:

$$
C_{\mathrm{o}}=\frac{1.5 L+5}{1-Y}
$$

where $C_{\mathrm{o}}$ - optimal cycle length (s); $L$ - total lost time (s); $Y$ - sum of critical phase flow ratios

The Webster cycle length model is used in the situations where the sum of flow ratios (the arrival flow to saturation flow ratio) is less than 1.0. However, this formulation has been proven to be ineffective oversaturated situations, and is inappropriate for other control type such as semi-actuated and full-actuated control.

For the sensitivity analysis, the two-level factorial design method was applied to Webster's optimal cycle length model given in Eq. (1). Total lost time $(L)$ and sum of critical phase flow ratios $(Y)$ could not be selected as individual parameters for the sensitivity analysis because they are dependent parameters. Total lost time changes with the number of the phases. An increase in the number of phase in a cycle length results in an increase of total lost time in a given cycle length. Sum of critical phase flow ratios is expressed as a function of both traffic flow $(q)$ and saturation flow $(s)$. Hence, Webster's optimal cycle length model defined in Eq. (2) can be rearranged as follows:

$$
C_{\mathrm{o}}=\frac{1.5 \cdot(n \cdot l)+5}{\left(1-\Sigma \frac{q}{s}\right)}
$$

where $C_{\mathrm{o}}$ - optimal cycle length (s); $n$ - the number of phase; $l$ - lost time in each phase (s); $q$ - traffic flow (vph/h); $s$ - saturation flow $(\mathrm{vph} / \mathrm{h})$.
Table 3 Lower and upper levels of model parameters used in the sensitivity analysis

\begin{tabular}{|c|c|c|c|c|}
\hline $\begin{array}{c}\text { Parameter } \\
\text { Index No }\end{array}$ & Parameter Name & Symbol & $\begin{array}{c}\text { Lower } \\
\text { Level }\end{array}$ & $\begin{array}{c}\text { Upper } \\
\text { Level }\end{array}$ \\
\hline 1 & Number of phases & $n$ & 2 & 5 \\
\hline 2 & Lost time (s) & $l$ & 2.5 & 4 \\
\hline 3 & Arrival flow (veh/h) & $q$ & 700 & 1200 \\
\hline 4 & Saturation flow (veh/h) & $s$ & 1500 & 1900 \\
\hline
\end{tabular}

Table 4 The design matrix of the single parameters

\begin{tabular}{|c|c|c|c|c|}
\hline & \multicolumn{5}{|c|}{ Model Parameters } \\
\hline Run & 1 & 2 & 3 & 4 \\
\hline 1 & - & - & - & - \\
\hline 2 & + & - & - & - \\
\hline 3 & - & + & - & - \\
\hline 4 & + & + & - & - \\
\hline 5 & - & - & + & - \\
\hline 6 & + & - & + & - \\
\hline 7 & - & + & + & - \\
\hline 8 & + & + & + & - \\
\hline 9 & - & - & - & + \\
\hline 10 & + & - & - & + \\
\hline 11 & - & + & - & + \\
\hline 12 & + & + & - & + \\
\hline 13 & - & - & + & + \\
\hline 14 & + & - & + & + \\
\hline 15 & - & + & + & + \\
\hline 16 & + & + & + & + \\
\hline
\end{tabular}

Four model parameters with parameter index numbers from 1 to $4(1: n, 2: l, 3: q, 4: s)$ were selected for the sensitivity analysis with the two-level factorial design method. The upper and lower levels of model parameters given in Tab. 3 were chosen arbitrarily within their reasonable ranges. For the given number of parameters and perturbation levels, the design matrix for the main parameters is given in Tab. 4 and the computation matrix for the multiple parameter interactions is given in Tab. 5 .

\begin{tabular}{|c|c|c|c|c|c|c|c|c|c|c|c|}
\hline \multirow[b]{2}{*}{ Run } & \multicolumn{11}{|c|}{ Multiple Parameter Interactions } \\
\hline & 12 & 13 & 14 & 23 & 24 & 34 & 123 & 124 & 134 & 234 & 1234 \\
\hline 1 & + & + & + & + & + & + & - & - & - & - & + \\
\hline 2 & - & - & - & + & + & + & + & + & + & - & - \\
\hline 3 & - & + & + & - & - & + & + & + & - & + & - \\
\hline 4 & + & - & - & - & - & + & - & - & + & + & + \\
\hline 5 & + & - & + & - & + & - & + & - & + & + & - \\
\hline 6 & - & + & - & - & + & - & - & + & - & + & + \\
\hline 7 & - & - & + & + & - & - & - & + & + & - & + \\
\hline 8 & + & + & - & + & - & - & + & - & - & - & - \\
\hline 9 & + & + & - & + & - & - & - & + & + & + & - \\
\hline 10 & - & - & + & + & - & - & + & - & - & + & + \\
\hline 11 & - & + & - & - & + & - & + & - & + & - & - \\
\hline 12 & + & - & + & - & + & - & - & + & - & - & + \\
\hline 13 & + & - & - & - & - & + & + & + & - & - & - \\
\hline 14 & - & + & + & - & - & + & - & - & + & - & - \\
\hline 15 & - & - & - & + & + & + & - & - & - & + & - \\
\hline 16 & + & + & + & + & + & + & + & + & + & + & + \\
\hline
\end{tabular}

Table 5 The computation matrix of the multiple parameter interactions

\section{SENSIVITY RESULTS OF THE WEBSTER'S OPTIMAL CYCLE LENGTH MODEL AND DISCUSSION}

For the given factorial design, 16 runs in total were carried out and the results for the optimal cycle length model are listed in Tab. 6. Additionally, the parameter effects for single and multiple parameter interactions are presented in the same table. To identify parameters with major effects on the model results, the parameter effects were plotted on a standard normal probability scale as suggested by Box et al. [1] (see Fig. 1).

As a single parameter effect, arrival flow on the model results has the highest effect when compared with other single parameters. As represented in the figure, the number of phase, saturation flow and the lost time in each phase were ranked as other single parameters, respectively. 
Similarly as two parameter interactions arrival flowsaturation flow $(q-s)$ were detected to be outliers which have major effects on the model results. For three parameter interactions, the effect of the number of the phase-arrival flow-saturation flow $(n-q-s)$ is slightly higher than the other three parameter interactions as seen in Tab. 6.

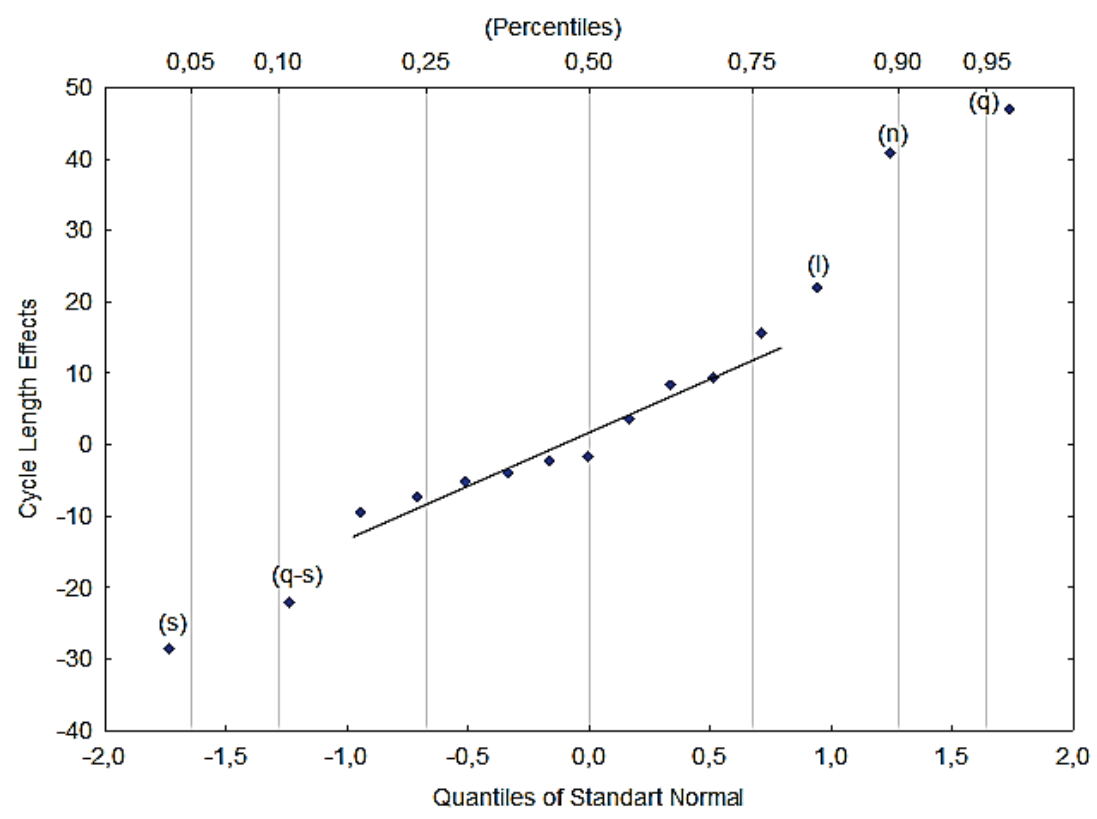

Figure 1 Parameter effects plotted on a normal probability scale

Table 6 Results of runs and parameter effects

\begin{tabular}{|c|c|c|c|}
\hline Run & $\begin{array}{c}\text { Optimal Cycle } \\
\text { Length }\end{array}$ & $\begin{array}{c}\text { Parameter } \\
\text { Index No }\end{array}$ & Parameter Effects \\
\hline 1 & 23.438 & 1 & 40.850 \\
\hline 2 & 44.531 & 2 & 21.996 \\
\hline 3 & 31.875 & 3 & 46.948 \\
\hline 4 & 65.625 & 4 & -28.432 \\
\hline 5 & 62.500 & 12 & 9.427 \\
\hline 6 & 118.750 & 13 & 15.561 \\
\hline 7 & 85.000 & 14 & -9.424 \\
\hline 8 & 175.000 & 23 & 8.379 \\
\hline 9 & 19.792 & 24 & -5.074 \\
\hline 10 & 37.604 & 34 & -21.997 \\
\hline 11 & 26.917 & 123 & 3.591 \\
\hline 12 & 55.417 & 124 & -2.175 \\
\hline 13 & 33.927 & 134 & -7.291 \\
\hline 14 & 64.464 & 234 & -3.926 \\
\hline 15 & 46.143 & 1234 & -1.682 \\
\hline 16 & 95.000 & & \\
\hline
\end{tabular}

As explained before, using the iterative method suggested by Henderson-Sellers, the model parameters were classified into two categories as primary and secondary importance. More specifically, the importance of these parameters was ranked on the absolute value of their effects at the four-, three-, and two-standard thresholds deviation (i.e. $4 \sigma, 3 \sigma$, and $2 \sigma$ ) as shown in Tab. 7.

Referring to Tabs. 6 and 7, and Fig. 1, arrival flow is of primary importance, while the remaining parameters are of secondary importance. This is not surprising because arrival flow is known as an effective parameter in determining traffic signal control strategies. As known the arrival flow to a pre-timed signalized intersection may vary from cycle to cycle due to stochastic nature of traffic. In some cycles, if arrival flow is greater than the capacity of a signalized intersection, the cycle will fail because of insufficient green time and cycle length. Thus, some vehicles have to wait the next cycle to be serviced.

Table 7 Importance of identified parameters based on thresholds of $|4 \sigma|,|3 \sigma|$

\begin{tabular}{|c|c|c|c|}
\hline \multirow{3}{*}{ Outliers } & $\begin{array}{c}\text { Primary } \\
\text { Importance }\end{array}$ & \multicolumn{2}{|c|}{ Secondary Importance } \\
\hline & $|4 \sigma|$ & $|3 \sigma|$ & $|2 \sigma|$ \\
\hline$q$ & $\checkmark$ & & \\
\hline$n$ & & $\checkmark$ & \\
\hline$l$ & & & $\checkmark$ \\
\hline$s$ & & & $\checkmark$ \\
\hline$q-s$ & & & \\
\hline
\end{tabular}

On the other hand, if arrival flow is less than the capacity some vehicles have to wait until the end of the cycle due to a fixed cycle length. Therefore, accurate estimation of arrival flow is important to determine the appropriate cycle length.

The analysis results indicate that, as main parameters, the number of phase in a cycle length, saturation flow and lost time have relatively lower effects on optimal cycle length as compared to arrival flow. The number of phase comes after arrival flow as another effective parameter on cycle length. This result is also expected because increasing numbers of phase in a cycle length results in larger delay, lost time and longer cycle length. The Webster's model proposes the use of a saturation flow which is the maximum rate of discharge and the lost time parameter. Sensitivity analysis results show that saturation flow is partly effective main parameter in the model. As known that saturation flow is defined as the number of vehicles per hour crossing signalized stop line if the signal remains green all the time. It is mainly depended on roadway and traffic conditions. While saturation flow is of primary importance for the capacity of intersection it is not so effective on cycle length, because it remains almost 
constant after the initial lost time has been accommodated Moreover, saturation flow depends on various factors such as lane with, number of lanes, grade, pedestrian activities etc. Lost time in the model appears to be the least effective main parameter in the examined cycle length model. The lost time is a portion of the cycle length which is not being fully employed. In other words, it is a time which is not effectively serving any traffic movements despite a green signal. Lost time almost remains fixed, regardless of cycle length, but shorter cycle lengths incorporates a large percentage of the lost time when compared to longer cycle lengths. According to sensitivity results, the interaction of arrival flow-saturation flow (q-s) seems to be relatively more effective on the selected cycle length model among the other two parameter interactions.

\section{CONCLUSION}

In this study, the sensitivity analysis of optimal cycle length model proposed by Webster and its parameters was investigated by utilizing the factorial design method. The results of the sensitivity analysis indicated that arrival flow among the main parameters has a significant effect on the optimal cycle length model. The study results also revealed that the remaining main parameters in the model (number of phase, saturation flow and lost time) have relatively lower effects on optimal cycle length. Additionally, the analysis results illustrated that the two-parameter interaction of arrival flow-saturation flow has a major effect on the model results. For the three-parameter interaction, the effect of the number of the phase-arrival flow-saturation flow has a slightly larger effect than the other three-parameter interactions but it was not a significant level. In summary, this study shows that the factorial design method is an effective way of examining the relative importance of the selected optimal cycle length model, and it can be employed for similar application in traffic engineering.

\section{REFERENCES}

[1] Box, G. E., Hunter, W. G., \& Hunter, J. S. (1978). Statistics for experimenters: an introduction to design, data analysis, and model building (Vol. 1). New York: Wiley.

[2] Henderson-Sellers, A. (1992). Assessing the Sensitivity of A Land-Surface Scheme to Parameters Used In TropicalDeforestation Experiments. Quarterly Journal of the Royal Meteorological Society, 118(508), 1101-1116. https://doi.org/10.1002/qj.49711850805

[3] Henderson-Sellers, A. (1993). A factorial assessment of the sensitivity of the BATS land-surface parameterization scheme. Journal of Climate, 6(2), 227-247. https://doi.org/10.1175/15200442(1993)006<0227:AFAOTS>2.0.CO;2

[4] Henderson-Sellers, B., \& Henderson-Sellers, A. (1996). Sensitivity evaluation of environmental models using fractional factorial experimentation. Ecological Modelling, 86(2), 291-295. https://doi.org/10.1016/0304-3800(95)00066-6

[5] Barros, A. P. (1996). An evaluation of model parameterizations of sediment pathways: a case study for the Tejo estuary. Continental Shelf Research, 16(13), 17251749. https://doi.org/10.1016/0278-4343(96)00009-X

[6] Akgüngör, A. P., Yildiz, O., \& Demirel, A. (2006). A Sensitivity Analysis of the HCM 2000 Delay Model with the
Factorial Design Method. Turkish Journal of Engineering and Environmental Sciences, 30(4), 259-267.

[7] Akgüngör, A. P., \& Yıldız, O. (2007). Sensitivity analysis of an accident prediction model by the fractional factorial method. Accident Analysis \& Prevention, 39(1), 63-68. https://doi.org/10.1016/j.aap.2006.06.013

[8] Akgüngör, A. P. (2011). The investigation of the parameter effects in Webster delay model with factorial design method. $9^{\text {th }}$ Transportation Congress / Istanbul, Turkey, May 16-18, 211-218 (in Turkish).

[9] Hatami, H., \& Aghayan, I. (2017). Traffic Efficiency Evaluation of Elliptical Roundabout Compared with Modern and Turbo Roundabouts Considering Traffic Signal Control. PROMET - Traffic \& Transportation, 29(1), 1-11. https://doi.org/10.7307/ptt.v29i1.2053

[10] Hao, Y., Teng, J., Wang, Y., \& Yang, X. (2016). Increasing Capacity of Intersections with Transit Priority. PROMET Traffic \& Transportation, 28(6), 627-637. https://doi.org/10.7307/ptt.v28i6.1999

[11] Nassiri, H., Tabatabaie, S., \& Sahebi, S. (2017). Delay-based Passenger Car Equivalent at Signalized Intersections in Iran. PROMET - Traffic \& Transportation, 29(2), 135-142. https://doi.org/10.7307/ptt.v29i2.2040

[12] Çalı̧̧kanelli, S. P., Coşkun Atasever, F., \& Tanyel, S. (2017). Start-up Lost Time and its Effect on Signalized Intersections in Turkey. PROMET - Traffic \& Transportation, 29(3), 321329. https://doi.org/10.7307/ptt.v29i3.2214

[13] Webster, F. V. (1958). Traffic signal settings, road research technical paper no. 39. Road Research Laboratory, London.

[14] Miller, A. J. (1968). Australian Road Capacity Guide: Provisional Introduction and Signalized Intersections. Australian Road Research Board, 4.

[15] Lan, C. (2004). New optimal cycle length formulation for pre-timed signals at isolated intersections. Journal of Transportation Engineering, 130(5), 637-647. https://doi.org/10.1061/(ASCE)0733-947X (2004) 130:5(637)

[16] Lan, C. J., \& Gu, X. (2005, September). Optimal signal controls and effects of flow uncertainty. In Intelligent Transportation Systems, 2005. Proceedings IEEE, 549-554.

[17] Cheng, D., Messer, C. J., Tian, Z. Z., \& Liu, J. (2003, January). Modification of Webster's minimum delay cycle length equation based on HCM 2000. In the $81^{\text {st }}$ Annual Meeting of the Transportation Research Board in Washington, DC.

[18] Cheng, D., Tian, Z. Z., \& Messer, C. J. (2005). Development of an improved cycle length model over the highway capacity manual 2000 quick estimation method. Journal of transportation engineering, 131(12), 890-897. https://doi.org/10.1061/(ASCE)0733-947X(2005)131:12(890)

\section{Contact information:}

\section{Ali Payıdar AKGÜNGÖR}

Department of Civil Engineering

Engineering Faculty, Kırıkkale University

Kırıkkale/Turkey

aakgungor@gmail.com

Ersin KORKMAZ (corresponding author)

Department of Civil Engineering

Engineering Faculty, Kırıkkale University

Kırıkkale/Turkey

ersin_korkmaz1@hotmail.com 